\title{
A Review of CFD Analysis of Heat Exchanger for Laminar Flow
}

\author{
Ravi Shankar Kumar \\ M. Tech Scholar \\ R.D. Engineering College \\ Ghaziabad, Uttar Pradesh, India \\ ravi632075@gmail.com
}

\author{
D.S. Chauhan \\ Assistant Professor \\ R.D. Engineering College \\ Ghaziabad, Uttar Pradesh, India \\ veerdharam76@gmail.com
}

\begin{abstract}
Helical coil heat exchangers, due to their maturity, are widely used in industrial applications such as the chemical and food industry, power generation, electronics, environmental technology, manufacturing industry, air conditioning, waste heat recovery, etc. on straight and cup heat exchangers. With its compact structure, larger heat transfer area and higher heat transfer capacity, etc., the twisted tap and classification of improvement techniques are presented in this paper. To present the dynamics, application, and advantages of CFD for computational fluids presented in this paper.
\end{abstract}

Keywords: CFD, Helical Coil, Heat Exchanger, Temperature.

\section{INTRODUCTION}

The process of improving the performance of a heat transfer system or increasing the heat transfer coefficient is known as increasing or improving heat transfer. This leads to a reduction in the size and cost of the heat exchanger. The increase in the heat transfer coefficient generally leads to a further advantage of reducing the driving force of the temperature, which increases the efficiency of the second law and reduces the generation of entropy. General techniques for improving heat transfer can be divided into three categories. The first is the passive method such as twisted tapes, spiral screw tape inserts, rough surfaces, elongated surfaces, and liquid and gas additives. The second method is the active method, which requires additional external energy, i.e. B. mechanical aids, vibrations of the surface fluid and use of electrostatic fields. Passive methods are cheaper than other groups. Twisted tape is one of the most important elements useful in laminar flow of this group. The third category involves the combined use of active and passive techniques to achieve a greater improvement in heat transfer than that produced by both when used individually, and is called composite enhancement. This technique is complex in design and therefore has limited applications. Techniques for increasing heat transfer (passive, active or a combination of passive and active methods) are commonly used in fields such as the process industry, heating and cooling in evaporators, thermal power plants, air conditioning systems, refrigerators, coolers for spacecraft and automobiles etc. exchanger. Also, as the heat exchanger ages, the resistance to heat transfer due to contamination or debris increases. These problems are more common with heat exchangers used in marine applications and the chemical industry. In some specific applications, such as B. Heat exchangers treating liquids with low thermal conductivity (gases and oils) and desalination plants, it is necessary to increase the heat transfer rate. The rate of heat transfer can be improved by interrupting the flow of fluid (by breaking viscous and thermal boundary layers), but this can greatly increase pumping capacity and ultimately increase the cost of pumping. Therefore, a desired heat transfer rate must be achieved in an existing heat exchanger with an economical pump capacity. Usually, vortex flow generators are placed in the flow channel to increase the heat transfer rate and this reduces the hydraulic diameter of the flow channel. The improved heat transfer in the pipe flow through inserts such as twist and screw belts is mainly due to flow blocking, flow splitting and secondary flow. Blocking the flow increases the pressure drop and leads to an increase in viscous effects due to the reduced free flow area.

\section{LITERATURE REVIEW}

Dheeraj Shriwas et al. [1] Heat exchangers are used in various industries to improve heat transfer. This article examines the different techniques used to improve heat transfer in order to discover new techniques for improving the rate of heat transfer.

Indri Yaningsih et al. [2] This article proposes a new design of a V-cut (VTT) twisted ribbon insert with a different aspect ratio (w/w), in which the heat transfer improved in the form of the 
properties of the Nusselt number $(\mathrm{Nu})$ and the friction factor (f) was investigated. Three different width ratios (weight / weight) 0.32 ; in this experiment, 0.38 and 0.48 are introduced. The working fluid is hot water in the inner tube and cold water in the annular space, the flow direction is counter-current. The incoming hot water temperature is kept constant at $60^{\circ} \mathrm{C}$ while the flow is in the Reynolds range \# 5400 - 17,350.

Tushar R. Shinde et al. [3] In this review, the focus is on research to improve heat transfer in heat exchanger tubes by using different types of twisted ribbon inserts with different twist angles, torsion ratios, shapes, etc., because twisted bands are one very popular tool for improving heat transfer. . The present work focuses on CFD and the experimental investigation of the heat transfer and friction factor of heat exchanger tubes equipped with perforated twisted tape inserts of different sizes.

Suhas V. Patil et al. [4] the present work provides an overview of the research work over the past decade to improve heat transfer in a circular tube and a square channel. In this thesis, the focus is on processing with twisted tape and screw tape inserts, as they are known, according to recent studies, as an economical tool in the field of heat transfer improvement.

Nimish Dubey et al. [5] in the present work, only a few design changes were made to a previously designed tube insert, and its heat output was analyzed using CFD software called ANSYS Fluent.

Shubham Jadhav et al. [6] this article introduces heat transfer techniques. Augmentation techniques refer to various methods of increasing the heat transfer rate without increasing size and without compromising overall performance. These techniques are used in heat exchangers. Some of the uses of heat exchangers are in the process industry, thermal power plants, air conditioning, spacecraft coolers, automobiles, etc. Therefore, heat exchangers are being developed for various applications that require careful analysis of the heat transfer rate and pressure drop estimates.

Orhan Keklikcioglu et al. [7] this article is a comprehensive review focusing on methods to improve heat transfer with wire wrapped and twisted ribbon inserts, as the inserts are easier and cheaper to install. The thermodynamic performance of the heat exchange components is also affected by flow conditions such as laminar or turbulent. This review includes studies to improve heat transfer using twisted webs and wire inserts wrapped in areas of laminar and turbulent flow.

\section{INSERTS}

Particular attention must be paid to operation to ensure fluid flow that improves and increases heat transfer.

The types of inserts are: twisted ribbon, wire spools, ribs, baffles, plates, spiral screw insert, mesh inserts, converging-diverging conical rings, conical rings etc. The corners of the wire mesh are considered the main techniques of the passive method; where twisted tape and threaded insert reel are more common than others. Twisted ribbons are metal bands that, depending on their shape and size, are twisted using some of the appropriate techniques and fed into the continuous flow to improve heat transfer. Twisted tape inserts are best and are often used in heat exchangers to improve heat transfer. Wire coil inserts have been used as one of the passive enhancement techniques and are widely used in heat transfer devices. We are studying the following uses: twisted ribbon, spools of thread.

\section{TWISTED TAPE}

Twisted faucets are metal tapes which, depending on their shape and size, are twisted and inserted into the flow using some of the appropriate techniques to improve heat transfer. Twisted tape inserts are best and are often used in heat exchangers to improve heat transfer.

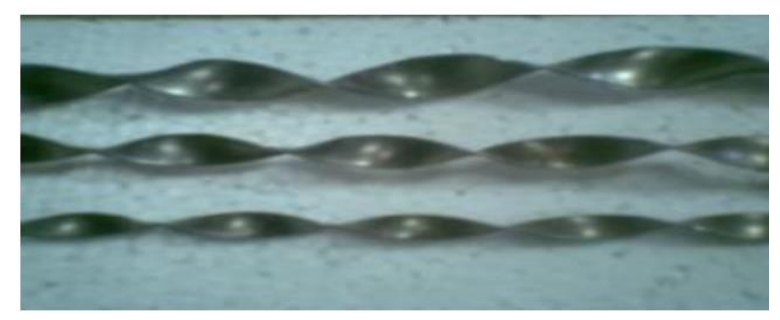

Fig. 1 Twisted Tape

Turned tap inserts increase heat transfer rates with a lower friction factor. The use of twisted ribbons in a tube provides a simple passive technique to improve convection heat transfer by creating a vortex in the strong flow, which breaks the boundary layer on the surface of the tube due to rapid changes in the geometry of the tube. of the tube. This means that these types of trajectories induce turbulence and eddy currents which are induced in the boundary layer and which give better results in terms of heat transfer coefficient and heat exchange due to changes in the geometry of the sheet metal inserts. At the same time, the pressure drop in the pipe increases when the twisted tape is used as an insert. To this end, many researchers have experimentally and numerically studied the desired design in order to obtain the best thermal performance with lower frictional losses. The improved heat transfer of twisted ribbon inserts is dependent on pitch and twist ratio. 


\section{ClassificAtion OF ENHANCEMENT TECHNIQUES}

Techniques for improving or increasing heat transfer consist in improving the thermo-hydraulic performance of heat exchangers. Existing improvement techniques can be broadly divided into three different categories:

\section{Passive Techniques \\ 2. Active Techniques \\ 3. Compound Techniques.}

\section{COMPUTATIONAL FLUID DYNAMICS}

Computational Fluid Dynamics (CFD) is the use of computeraided simulation to analyze systems that affect fluid flow, heat transfer and related phenomena such as chemical reactions [8]. A digital model is first built using a series of mathematical equations that describe flow. These equations are then solved using a computer program to obtain the flow variables over the entire flow range. Since the advent of the digital computer, CFD has received a lot of attention and has been widely used to study various aspects of fluid dynamics. The development and application of CFDs has experienced significant growth and has therefore become a powerful tool for the design and analysis of engineering and other processes. In the early 1980s, computers became so powerful that generic CFD software became available.

\section{APPLICATIONS AND ADVANTAGES OF CFD}

Numerous developments in the field of CFD, coupled with its ability to model complex phenomena and the growing popularity of such software, have broadened the reach of CFD. It is used in a wide variety of industries, including mechanical, process, petroleum, energy, metallurgical, biomedical, pharmaceutical and food industries. CFD techniques have been widely used in the process industry to better understand different flow phenomena, study different device designs or compare performance under different operating conditions. Examples of CFD applications in the chemical process industry include drying, combustion, separation, heat exchange, mass transfer, pipeline flow, reaction, mixing, multiphase systems and material processing. CFD has also been applied to a number of food processing operations such as drying, cooling, sterilization, blending and heat exchangers. CFD has also been used successfully in modeling various multiphase flow systems, particularly gas-solid mixtures, although some limitations still exist. Multi-phase CFD models can help understand the 14 complex interactions between the different phases and provide detailed transient 3D information that experimental approaches may not be able to provide. These applications show, among other things, the potential of CFD to simulate complex processes and thus the possibility of examining a broader spectrum of processes.

\section{CONCLUSION}

Conventional methods of designing and developing heat exchangers are expensive. CFD offers an alternative to a quick and cost-effective solution for the design and optimization of heat exchangers. CFD results are an integral part of the design process and eliminate the need for a prototype. Presentation of the dynamics, application and advantages of CFD for the calculation of fluids, which are presented in this article. Twist tap and classification of improvement techniques are presented. New flow modeling strategies can be developed for simulating flow in a tube and laminar flow tube heat exchanger.

\section{REFERENCES}

[1] Dheeraj Shriwas, Mr. Jagdeesh Sain "Heat Transfer Enhancement Technique in Heat Exchanger: An Overview", 2018 IJRTI | Volume 3, Issue 9 | ISSN: 2456-3315.

[2] Indri Yaningsih, Agung Tri Wijayanta "V-cut Twisted Tape Insert Effect on Heat Transfer Enhancement of Single Phase Turbulent Flow Heat Exchanger", AIP Conference Proceedings 1931, 030038 (2018); https://doi.org/10.1063/1.5024097.

[3] Tushar R. Shinde, Prof. D. G. Kumbhar "A Review on Experimental and CFD Analysis for Heat Transfer Enhancement in Heat Exchanger Tube using Drilled Twisted Tape Inserts of various Geometries", International Research Journal of Engineering and Technology (IRJET), Volume: 04 Issue: 06 | June 2017.

[4] Suhas V. Patil and P. V.Vijay Babu "Heat Transfer Augmentation in a Circular tube and Square duct Fitted with Swirl Flow Generators: A Review", International Journal of Chemical Engineering and Applications, Vol. 2 , No. 5 , October 2011.

[5] Nimish Dubey, Morrish Kumar "CFD Analysis of Fluid Flowing Through a Heat Exchanger Tube Having a Twisted Tape with a Centrally Placed Semi-Circular Groove", International Journal of Science and Research (IJSR), Volume 6 Issue 6, June 2017.

[6] Shubham Jadhav, Jayesh Jain "Heat Transfer Augmentation Technique Using Twisted Tape Insert", International Conference on Ideas, Impact and 
Innovation in Mechanical Engineering (ICIIIME 2017), Volume: 5 Issue: 62017.

[7] Orhan Keklikcioglu, and Veysel Ozceyhan "A Review of Heat Transfer Enhancement Methods Using Coiled Wire and Twisted Tape Inserts", 2018.

[8] Versteg H. and Malasekara W, An Introduction to Computational Fluid Dynamics, (2nd edition, year of publication-2007), Pearson Publications. 